
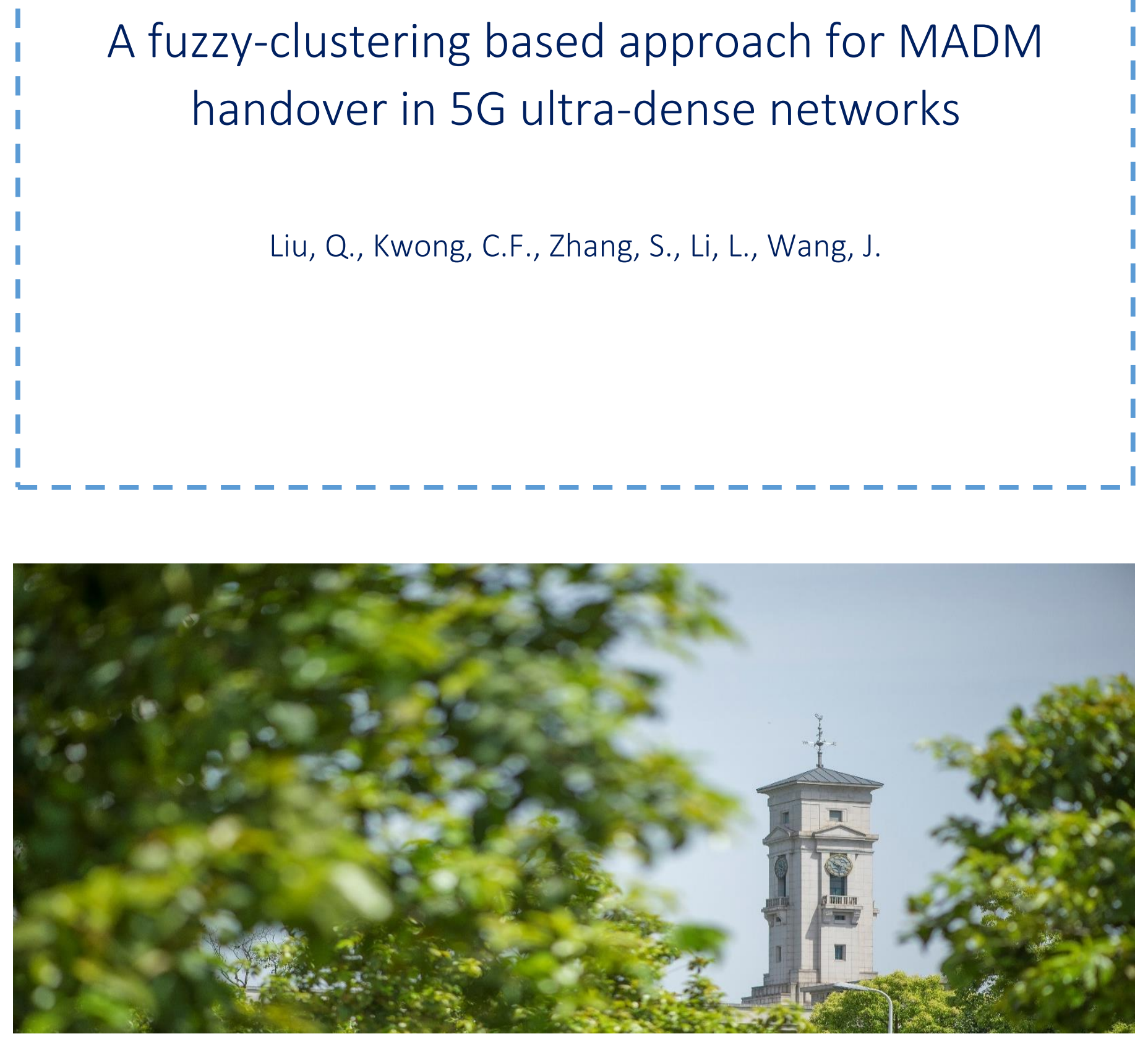
University of Nottingham Ningbo China, 199 Taikang East Road, Ningbo, 315100, China

First published 2019

This work is made available under the terms of the Creative Commons Attribution 4.0 International License:

http://creativecommons.org/licenses/by/4.0

The work is licenced to the University of Nottingham Ningbo China under the Global University Publication Licence:

https://www.nottingham.edu.cn/en/library/documents/researchsupport/global-university-publications-licence.pdf Nottingham 


\title{
A fuzzy-clustering based approach for MADM handover in 5G Ultra-Dense Networks
}

\author{
Qianyu Liu ${ }^{1}$, Chiew Foong Kwong, ${ }^{2, *}$, Sibo Zhang ${ }^{2}$, Lincan Li ${ }^{2}$ and Jing Wang ${ }^{2}$ \\ ${ }^{1}$ University of Nottingham Ningbo China, International Doctoral Innovation Centre, Ningbo, China \\ Qianyu.Liu@nottingham.edu.cn \\ ${ }^{2}$ University of Nottingham Ningbo China, Department of Electrical and Electronic, Ningbo, China \\ \{c.f.Kwong, zy18533, Lincan.Li, Jing.Wang\}@nottingham.edu.cn
}

\begin{abstract}
As the global data traffic has significantly increased in the recent year, the ultra-dense deployment of cellular networks (UDN) is being proposed as one of the key technologies in the fifthgeneration mobile communications system $(5 \mathrm{G})$ to provide a much higher density of radio resource. The densification of small base stations (BSs) could introduce much higher inter-cell interference and lead user to meet the edge of coverage more frequently. As the current handover scheme was originally proposed for macro BS, it could cause serious handover issues in UDN i.e. ping-pong handover, handover failures and frequent handover. In order to address these handover challenges and provide a high quality of service (QoS) to the user in UDN. This paper proposed a novel handover scheme, which integrates both advantages of fuzzy logic and multiple attributes decision algorithms (MADM) to ensure handover process be triggered at the right time and connection be switched to the optimal neighbouring BS. To further enhance the performance of the proposed scheme, this paper also adopts the subtractive clustering technique by using historical data to define the optimal membership functions within the fuzzy system. Performance results show that the proposed handover scheme outperforms traditional approaches and can significantly minimise the number of handovers and the ping-pong handover while maintaining QoS at a relatively high level.
\end{abstract}

Keywords: mobility management, handover, fuzzy logic, MADM, fuzzy-TOPSIS, ultradense networks (UDNs), subtractive clustering, 5G

\section{Introduction}

The global mobile data traffic has significantly increased in recent year. As estimated by Cisco [1], the monthly global mobile data traffic will be 77 exabytes by 2022, and annual traffic will reach almost one zettabyte. The fifth generation of mobile communications (5G) will implement ultra-dense network (UDN) to cope with this demand. The UDN is achieved by deploying a massive number of small base 
stations (BS) within traditional macro BSs's coverage. Under this architecture, a more substantial amount of simultaneous mobile data can be delivered by the small BSs, which could offload part of the data traffic from macro BSs. Therefore, the radio resources management (RRM) can be more efficient, and the system capacity is significantly improved as compared to the macro stations in 4G. When user equipment (UE) moving within cellular networks, the UE will change its serving BS to maintain its connection through handover. At present, the handover scheme in 4G [2] and 5G [3] is mainly proposed for macro BS systems and triggered by the $A 3$ event. In the A3 event, the user equipment (UE) will request a handover when one of the RRM decision attributes at the neighbouring BS, such as reference signal receiving power (RSRP) or signal to interference and noise ratio (SINR) is superior to the serving BS. This triggering mechanism has the advantage of lower operational costs and complexity.

However, the small BSs in UDN are deployed denser and closer to each other. Consequently, it introduces higher inter-cell interference, which leads to mobile user to meet the edge of coverages more frequently. As the A3 event is a single attribute algorithm and proposed for macro BS system, which can be easily affected by fluctuation of the RSRP due to interference. The existing handover scheme in UDN can frequently trigger the handover process even with a slightest physical movement of UE. The data flow will also be often interrupted as the handover process will first break the serving link before re-establishing the link to the target BSs. Subsequently, the system capacity gain that obtained by network densification is diminished by frequent handovers. The frequent handovers can also cause higher core networks signalling load and overall latencies that are contributing to the degradation in Quality of Service (QoS). Furthermore, single attribute operations of A3 event does not sufficiently consider other attributes that will affect the QoS in UDN such as jitter, packet loss, latencies, etc. By implementing an existing handover scheme of macro BS at UDN could result in poor system performance and QoS.

To address these challenges, the triggering of handovers needs to be at the exact right time, and UE's connection needs to be transferred to the most suitable target BS. There are many previous studies on fuzzy logic based handover triggering mechanism to allow both timely and flexibility to start a handover process [4]-[7]. Fuzzy logic is a reliable and mathematically robust tool to process uncertainty data within the mobile environment and conduct multivariate analysis. In [4], the authors proposed a fuzzy logicbased triggering scheme in UDN that considers user velocity and radio channel quality to adapts hysteresis margins of A3 event. In [5], a context-aware fuzzy handover scheme is proposed to minimise frequent handover problem by adopting multiple user context parameters such as velocity, SINR, throughput and 
BSs load. In [6], three fuzzy logic controller that can define handover hysteresis was developed to different cell outage failures. Reference [7] develops a hybrid fuzzy logic-based decision algorithm. First, the algorithm predicts the received signal strength by using an artificial neural networks-based model. Then other measured network parameters is used together with the predicted received signal strength as the input of fuzzy logic to trigger the handover process.

The simulation results in [4]-[7] demonstrates the fuzzy logic based triggering mechanism can effectively minimise ping-pong handover effect, call drops ratio, and at the same time maximises the throughput. However, conventional fuzzy logic has its flaws. For instance, it cannot produce a reliable decision when there are too many decision criteria as the input of the fuzzy inference engine. Also, the design of the membership function needs to rely heavily on the human experience, and considerable effort in tuning for optimisation. These works did not elaborate further on how the membership functions are optimised for each criterion. Therefore, the reliability of these approaches cannot be guaranteed in different application scenarios. In this situation, artificial intelligence is seen to be a useful tool to define fuzzy membership functions for its strong capability in statistical analysis, decision making, pattern recognition, etc. [8]-[12]. Reference [13] shows the adaptive handover triggering mechanisms by combining fuzzy logic with neuro networks. In this approach, the subtractive clustering is used to initialise membership functions, which is tuned by using artificial neuro network corresponds to the changes in the mobile environment. The result shows that the proposed adaptive fuzzy controller can effectivity generate and adjust fuzzy membership function, which can reduce the number of handovers, ping-pong ratio, and call drop ratio effectively in the different application scenarios. However, this approach cannot deal with too many inputs as it will compromise the reliability of the algorithm.

Apart from triggering handover process at the right time, the targeted BS needs to be carefully selected to ensure guaranteed QoS. At present, this is usually done by using the multiple attributes decision making (MADM) approach. The MADM is a mathematical tool to deal with decision-making problems with multiple conflicting attributes. There are many types of MADM methods, and among the most popular variant is the techniques for order preference by similarity for an ideal solution (TOPSIS). TOPSIS is favoured due to its robustness and reliability, as discussed in surveys [14]-[16]. The TOPSIS approach was first developed by Hwang and Yoon [17] and is widely used for target BS selection, as reported in [18]-[22]. References [18] and [19] proposed a MADM based handover scheme to eliminate unnecessary handover in LTE-Advance and UDN. The proposed scheme first applies a subjective weighting approach 
- analytical hierarchy process mechanism to prioritise the criteria for obtaining the weight. Then TOPSIS engine is implemented to select the best access networks amongst all candidates. Reference [20] proposed two novel TOPSIS-based handover algorithms in ultra-dense heterogeneous networks by using two different objective weighting approach entropy and standard deviation weighting techniques. These two weighting approaches can effectively avoid errors caused by human subjective decisions. Research works in [21] and [22] demonstrated an enhanced TOPSIS based handover scheme by combining TOPSIS with other MADM schemes, i.e. utility function and analytical hierarchy process. The performance of each access point technologies based on the traffic class is first evaluated by analytical hierarchy process and TOPSIS decision engine. Subsequently, the utility function is then implemented to represent the desires of the users on the traffic class for optimal network selection.

Simulation results in [18]-[22] shows that TOPSIS approach can effectivity deal with multiple handover related criteria and select an optimal handover target, which can significantly reduce ping-pong effect, number of handover failures, and enhancing user throughput. However, conventional MADM approach cannot deal directly with any form of imprecise data [23]. When random radio signal fluctuation occurs in the input sequence of MADM, the selected target BS is usually not an optimal option. In this situation, fuzzy logic is useful tools to process uncertain information by combing with MADM approach. Research work [24] shows a general view of fuzzy-TOPSIS and [25] conducts a comparison between conventional TOPSIS and fuzzy-TOPSIS. The result shows that for the input parameter with fluctuations and unpredictable errors, the fuzzy-TOPSIS will make a better decision than the conventional TOPSIS. Given the situation where the parameters such as RSRP, SINR and Jitter are frequently experiencing rapid fluctuations, the fuzzy-TOPSIS is seen to be an excellent choice to support handover decision-making. Reference [26] shows a cell-selection scheme based on fuzzy-TOPSIS in LTE. The simulation result shows that the fuzzy-TOPSIS approach can outperform the conventional MADM approach in terms of ping-pong handover, number of handover and handover failure ratio. However, the method described in [26] is using generalised membership functions in the fuzzification process. Thus the algorithm is potentially unreliable. Also, it proposed only cell selection schemes while ignoring the triggering process of handover.

Due to the aforementioned challenges in UDN that cannot be effortlessly addressed by existing approaches. In this paper, we solve the handover problem in UDN with objectives of minimising the number of handovers, ping-pong effect, and providing high QoS to the user, by using a fuzzy-TOPSIS 
decision model with clustering technique. Specifically, the proposed handover scheme in this paper can both decide a suitable triggering timing and select the optimal target selection scheme by considering multiple measured network parameters.

The main contributions of this paper are summarised follows:

- Firstly, we integrate both advantages of fuzzy logic and TOPSIS into handover scheme to select the most suitable target BS. Specifically, the fuzzy set is implemented to process multiple fluctuated network parameters as input if TOPSIS, due to its capability to deal with uncertain data. The TOPSIS decision engine will select an optimal neighbouring BS as the handover target as it can efficiently deal with multiple conflict attribute.

- Secondly, we adopt the outcome of TOPSIS decision engine - closeness coefficient (CC) to determine triggering timing. This triggering mechanism allows BS to decide a triggering timing based on the overall performance of the application scenario rather than the RRM condition of UE's serving BS.

- Thirdly, we utilise an objective weighting to calculate weight value for each attribute based on information entropy. By this approach, the subjective error caused by human experience can hence be eliminated.

- Finally, in order to further enhance the performance of the proposed scheme. We adopt the subtractive clustering techniques to generate fuzzy membership functions by using historical data. With the strong statistical analysis ability of subtractive clustering, the proposed scheme can intelligently decide the most suitable fuzzy membership function for different handover criteria. To the best of authors' knowledge, this is the first works that applied clustering techniques into fuzzy TOPSIS decision engine.

The rest of this paper is organised as follows: The preliminaries about fuzzy sets and TOPSIS are introduced in Section 2. The comprehensive methodology of fuzzy clustering based MADM handover scheme is presented at section 3 , with the subtractive clustering method to generate fuzzy membership function in section 3.1, the generation of decision matrix in section 3.2, the decision-making process by TOPSIS in section 3.3, and the triggering mechanism and target BS selection scheme in section 3.4. The performance of the proposed algorithm will be evaluated in a simulation environment and compared with the conventional handover algorithm in Section 4. Finally, the paper will be concluded in Section 5. 


\section{Preliminary}

Before proceeding to introduce the proposed scheme, we first describe some essential fundamentals in this section, i.e., the fuzzy set and decision matrix of MADM, where the max-min normalisation and entropy approaches are introduced, which will be utilised to support decision making. Finally, the framework of TOPSIS decision engine is presented, which will be integrated with fuzzy logic and implemented to making decisions in triggering and handover target selection.

\section{$2.1 \quad$ Fuzzy sets}

Fuzzy sets are a class of elements with continuous values of membership (Fig.1). For each fuzzy set, a membership function is used to assign a grade of membership to each of the objects [4]. The work described in this paper, triangular fuzzy membership functions are used for its simplicity and computation efficiency.

As expressed in Eq. (1), a triangular fuzzy number, $\tilde{a}$, in a universal set $X$ is described by a membership function $\mu_{\tilde{a}}(x)$ that associates each element $x$ in $X$ with a grade of membership range [0,1]. The value of $\mu_{\tilde{a}}(x)$ is the membership grade of $x$ in $\tilde{a}$. From (1), $a_{1}$ and $a_{3}$ are the lower and upper bounds of the fuzzy number $\tilde{a}$, and $a_{2}$ is the modal value for $\tilde{a}$.

$$
\mu_{\tilde{a}}(x)=\left\{\begin{array}{c}
0, x \leq a_{1} \\
\frac{x-a_{1}}{a_{2}-a_{1}}, a_{1}<x \leq a_{2} \\
\frac{a_{3}-x}{a_{3}-a_{2}}, a_{2}<x \leq a_{3} \\
0, x>3
\end{array}\right.
$$

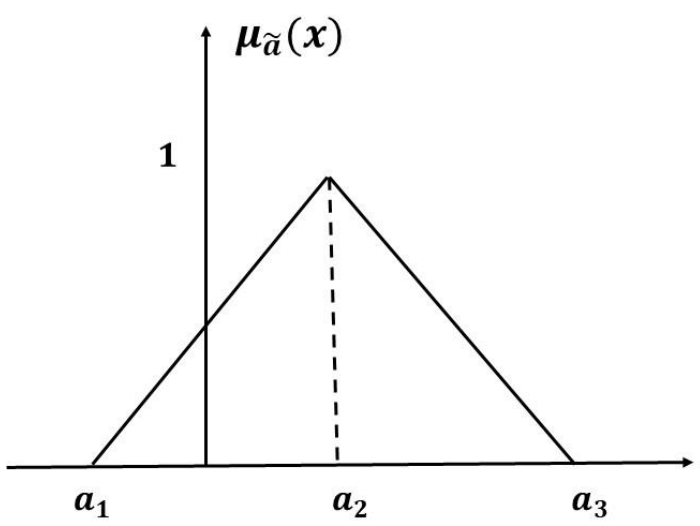

Fig. 1 The membership functions of a triangular fuzzy number 


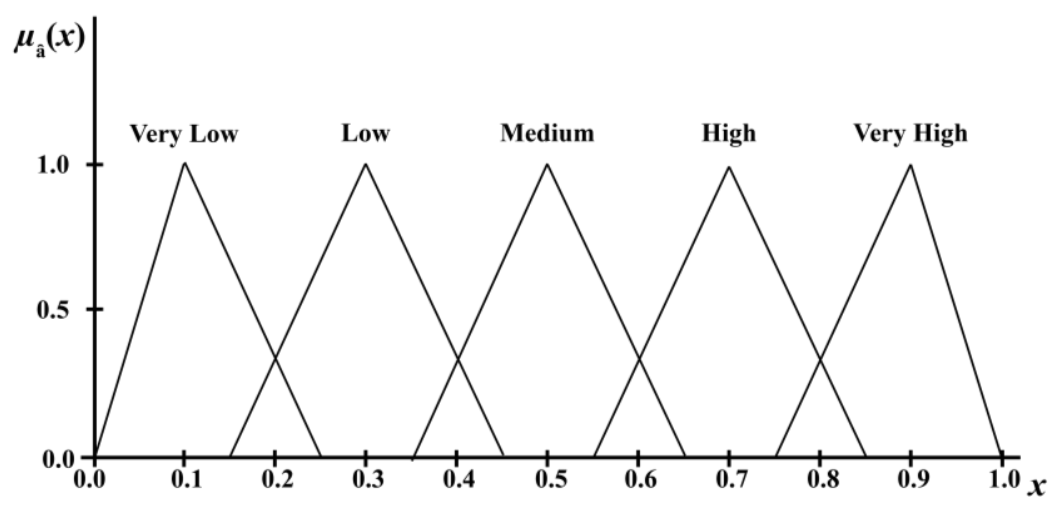

Fig 2. Fuzzy triangular membership functions

Different fuzzy sets are often labelled by linguistic variables to associate them with the imprecise terms appear in human's language. For instance, in Fig.2, five triangular membership functions are associated with different linguistic variables which are very low, low, medium, high and very high. Let $\tilde{a}=$ $\left(a_{1}, a_{2}, a_{3}\right)$ and $\tilde{b}=\left(b_{1}, b_{2}, b_{3}\right)$ be two non-negative triangular fuzzy numbers. According to the extension principle, the arithmetic operations are defined as follows [24]:

$$
\begin{gathered}
\tilde{a}+\tilde{b}=\left(a_{1}, a_{2}, a_{3}\right)+\left(b_{1}, b_{2}, b_{3}\right)=\left(a_{1}+b_{1}, a_{2}+b_{2}, a_{3}+b_{3}\right) \\
\tilde{a}-\tilde{b}=\left(a_{1}, a_{2}, a_{3}\right)-\left(b_{1}, b_{2}, b_{3}\right)=\left(a_{1}-b_{1}, a_{2}-b_{2}, a_{3}-b_{3}\right) \\
k \tilde{a}=\left(k a_{1}, k a_{2}, k a_{3}\right) \\
\tilde{a} \times \tilde{b}=\left(a_{1}, a_{2}, a_{3}\right) \times\left(b_{1}, b_{2}, b_{3}\right)=\left(a_{1} \times b_{1}, a_{2} \times b_{2}, a_{3} \times b_{3}\right)
\end{gathered}
$$

The distance between $\tilde{a}$ and $\tilde{b}$ cam be calculated in Eq. (6):

$$
d(\tilde{a}, \tilde{b})=\sqrt{\frac{1}{3}\left[\left(a_{1}-b_{1}\right)^{2}+\left(a_{2}-b_{2}\right)^{2}+\left(a_{3}-b_{3}\right)^{2}\right]}
$$

\subsection{Decision Matrix for MADM}

The first step of most of MADM techniques is to construct a decision matrix. Given $m$ alternatives, $A=$ $\left\{A_{i} \mid k=1, \ldots, m\right\}$, and each alternatives have $n$ attribute, $C=\left\{C_{j} \mid j=1, \ldots, n\right\}$, a decision matrix $D M=$ $\left\{x_{i j} \mid k=1, \ldots, m ; j=1, \ldots, n\right\}$ can be formed as $(7)$

$$
\begin{array}{ccccccc}
\multicolumn{1}{c}{C_{1}} & C_{2} & C_{3} & \ldots & \ldots & C_{n} \\
\multicolumn{1}{c}{A_{1}} & x_{11} & x_{12} & x_{13} & \ldots & \ldots & x_{1 n} \\
A_{2} & x_{21} & x_{22} & x_{23} & \ldots & \ldots & x_{2 n} \\
\vdots & \vdots & \vdots & \vdots & \ddots & \ddots & \vdots \\
\vdots & \vdots & \vdots & \vdots & \ddots & \ddots & \vdots \\
A_{m} & x_{m 1} & x_{m 2} & x_{m 3} & \ldots & \ldots & x_{m n}
\end{array}
$$


Where, $x_{i j}$ in $D M$ represents the attribute $C_{j}(j$ from 1 to $n)$ of the alternatives $A_{i}(i$ from 1 to $m$ ). For instance, in this work, the alternatives $A_{1}$ represents candidate BS - $A_{1}$ have $n$ decision attribute from $x_{11}$ to $x_{1 \mathrm{n}}$.

As each criterion in the matrix are often measured in different units, and hence the normalisation process is necessary to avoid ambiguity. In this work, the Min-Max scaling approach will be adopted to normalise all benefit (Eq. 8) and cost (Eq.9) criteria of DM.

$$
\begin{aligned}
Z_{i j} & =\frac{\left[x_{i j}-\min \left\{x_{i j}\right\}\right]}{\left[\max \left\{x_{i j}\right\}-\min \left\{x_{i j}\right\}\right]} \\
Z_{i j} & =\frac{\left[\max \left\{x_{i j}\right\}-x_{i j}\right]}{\left[\max \left\{x_{i j}\right\}-\min \left\{x_{i j}\right\}\right]}
\end{aligned}
$$

\subsection{TOPSIS decision engine}

The core idea of TOPSIS is to select the optimal alternative which closest to the positive-ideal solution (PIS) as well as farthest to the negative-ideal solution (NIS), which calculated as:

$$
\begin{aligned}
& P I S=\left\{v_{j}^{+} \mid j=1,2, \ldots, n\right\}=\left\{\left(\max _{i} v_{i j} \mid i=1,2, \ldots, m\right) \mid j=1,2, \ldots, n\right\} \\
& N I S=\left\{v_{j}^{-} \mid j=1,2, \ldots, n\right\}=\left\{\left(\min _{i} v_{i j} \mid i=1,2, \ldots, m\right) \mid j=1,2, \ldots, n\right\}
\end{aligned}
$$

Where $v_{i j}$ is weighted normalised decision matrix derived by multiplying normalised decision matrix with the weight value.

The next step is to measure the distance from the PIS and the NIS to each candidate. This can be achieved by using Euclidean distance:

$$
\begin{aligned}
& D_{i}^{+}=\sqrt{\sum_{j=1}^{m}\left(v_{i j}-v_{j}^{+}\right)^{2}}, i=1, \ldots, m \\
& D_{i}^{-}=\sqrt{\sum_{j=1}^{m}\left(v_{i j}-v_{j}^{-}\right)^{2}}, i=1, \ldots, m
\end{aligned}
$$

The closeness coefficient of similarities to the PIS can then be computed as:

$$
C C_{i}=\frac{D_{i}^{-}}{D_{i}^{+}+D_{i}^{-}}
$$

where $C C_{i} \in[0,1] \quad \forall i=1, \ldots, m$. The order of preference can be obtained with respect to $C C_{i}$ in descending order and the best alternative can hence be selected. 


\section{Methodology}

Algorithm 1 shows the detailed procedure of the proposed fuzzy clustering-based approach for MADM handover. Generally, the scheme has three main parts. Firstly, in the initialisation stage, the historical data of handover criteria will be processed by the subtractive clustering technique to generate fuzzy membership function for each criterion. This will be explained in detail in Section 3.1. And the handover criteria measured by UE will be fed into the decision matrix, which will be explained in Section 3.2. In the next stage, the fuzzy membership function and decision matrix will be processed by fuzzy TOPSIS decision engine (see Section 3.3). The output of the decision engine will be used to determine the handover triggering timing and target BS in the last stage (see Section 3.4).

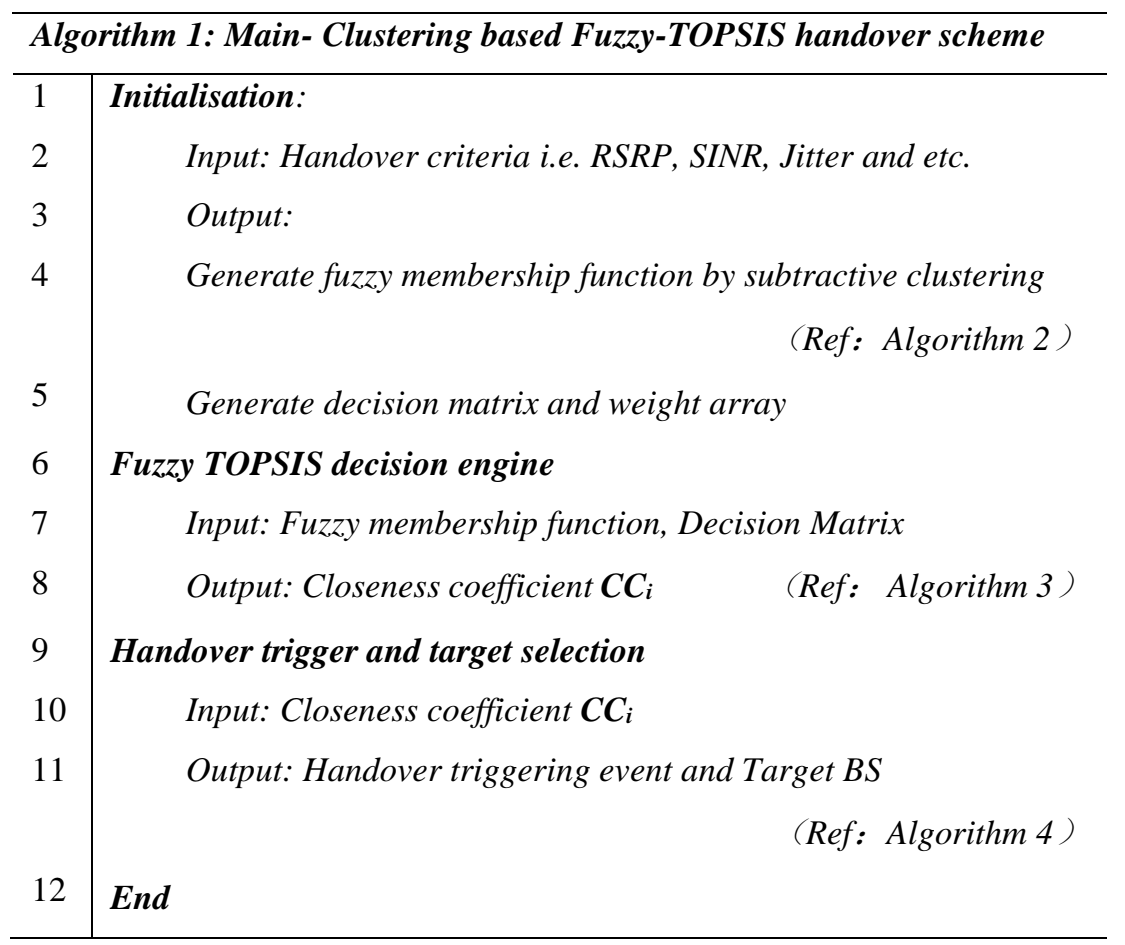

\subsection{Initialisation - Subtractive clustering method to generate fuzzy membership function}

The fuzzy membership functions play an essential role at fuzzy-TOPSIS decision engine, which can easily affect the performance of the proposed scheme. An effective membership functions need to fit the pattern of the probability distribution of its input data, which in turn can reflect the actual level of data (i.e. low, medium, high, etc.). Most of the fuzzy-TOPSIS based algorithms in the previous work are using fuzzy membership functions that are distributed evenly. This arrangement will only be reasonable if the 
probability distribution of the input data is uniformly distributed, where a randomly selected data point is expected to have the same chance of being allocated to each fuzzy set.

In practice, the input data of fuzzy-TOPSIS can follow various patterns of the probability distribution, and thus the membership functions for different input parameter should be adjusted to fit in correspondingly. Therefore, the design of the membership function requires statistics analysis to the collected data. As mentioned earlier, we adopt the subtractive clustering method to define membership function from historical data without going through the conventional process of trial and error. The clustering algorithm can locate the concentration inside the historical data to identify the centres and spans of the fuzzy membership functions. Among all types of clustering algorithms, the subtractive clustering is selected in the proposed scheme due to its "one-pass" method, which ultimately contributes to high computational efficiency. This approach will also allow the algorithm to obtain an optimal decision from MADM.

Given that $n$ data sets measured in $m$-dimensional input space

$$
\left\{\overrightarrow{\boldsymbol{x}_{\boldsymbol{\imath}}}=\left(x_{i 1}, x_{i 2}, \ldots, x_{i m}\right) \mid i \in[1, n]\right\}
$$

Each data set is a vector consisting of $m$ values corresponding to the $m$ dimensions, which represents $m$ attributes used in MADM methods.

The subtractive clustering initiates by evaluating the potential of each data set to be the cluster centres.

The potential value of $\overrightarrow{\boldsymbol{x}_{\boldsymbol{i}}}$ is calculated as

$$
\begin{gathered}
P_{i}=\sum_{j=1}^{n} e^{-\alpha\left\|\overrightarrow{\boldsymbol{x}_{\boldsymbol{\imath}}}-\overrightarrow{\boldsymbol{x}_{j}}\right\|^{2}} \\
\alpha=\frac{4}{r_{a}^{2}}
\end{gathered}
$$

$r_{a}$ is the range of influence and defines a local neighbourhood where the concentration to be considered. The data point assigned with the highest potential value is treated as the first data clustering centre. Let $\overrightarrow{\boldsymbol{x}}_{\mathbf{1}}$ ' be the location of the first cluster centre and $P_{1}^{*}$ be the potential of it. The potential values for the data points are then updated as

$$
\begin{gathered}
P_{i} \leftarrow P_{i}-P_{1}^{*} e^{-\beta\left\|\overrightarrow{\boldsymbol{x}_{\boldsymbol{i}}}-{\overrightarrow{\boldsymbol{x}_{1}}}^{*}\right\|^{2}} \\
\beta=\frac{4}{r_{b}^{2}}
\end{gathered}
$$




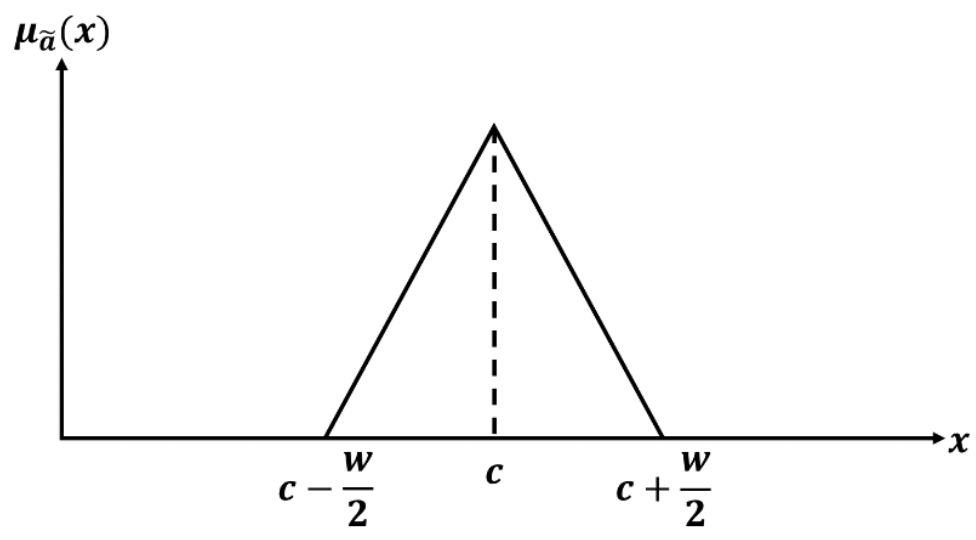

Fig. 3. Parameters to plot triangular membership function

The potential values $P_{i}$ of all data points are decreased by a factor, $e^{-\beta\left\|\overrightarrow{x_{i}}-\vec{x}_{x_{1}}^{*}\right\|^{2}}$, which is a function of the distance from each data point to ${\overrightarrow{\boldsymbol{x}_{1}}}^{*}$. If one point is close to ${\overrightarrow{\boldsymbol{x}_{\mathbf{1}}}}^{*}$, its potential will be greatly reduced and hence it is unlikely to be selected as the next clustering centre. The radius of the range is defined by $r_{b}$ where the potential will be largely reduced. The setting of $r_{b}$ and $\beta$ controls the space between cluster centres.

The data that has the highest updated potential, $P_{i}$ will be chosen as the next cluster centre. The potential values of all the data points will then be further reduced. In general, after the location of $k$ th clustering centre is found, the potential value of all the points are revised as,

$$
P_{i} \leftarrow P_{i}-P_{k}^{*} e^{-\beta\left\|{\overrightarrow{x_{l}}}_{-}{\overrightarrow{x_{k}}}^{*}\right\|^{2}}
$$

where ${\overrightarrow{\boldsymbol{x}_{\boldsymbol{k}}}}^{*}$ is the $k$ th data centre and $P_{k}^{*}$ is the potential value of it.

The process of obtaining a new clustering centre and updating the potential values repeats until $P_{k}^{*}<$ $\varepsilon P_{1}^{*}$, where $\varepsilon$ is the rejection ratio. Hence subtractive clustering stops when the potential of the last acquired centre is too weak to be the next clustering centre.

The triangular membership functions generated by subtractive clustering are defined by the centre, $c$, and width, $w$, as depicted in Fig.3. To convert the obtained clusters into fuzzy membership functions, the location the cluster centres $\left\{\overrightarrow{\boldsymbol{x}}_{\boldsymbol{\imath}}^{*}=\left(x_{i 1}, x_{i 2}, \ldots, x_{i m}\right)\right\}$ are equivalent to the centre membership functions. For example, the centres of membership functions for the $\mathrm{j}$ dimension are $\left\{x_{1 j}, x_{2 j}, x_{3 j}, \ldots \ldots\right\}$. The width $w_{j}$ is calculated as,

$$
w_{j}=d\left(U_{j, \max }-U_{j, \min }\right)
$$


where $U_{j, \max }$ and $U_{j, \min }$ are the maximum and minimum of values of the data set in the $j$ th dimension. The value of constant $d$ can be adjusted manually and will not have any significant effects to the result due to the nature of fuzzy-TOPSIS that the distances of each membership are mainly determined by $c$. However, it is essential to assure that the whole input space is under the coverage of the generated triangular membership functions.

The parameters involved in subtractive clustering were set to be $\{\alpha=16, \beta=12, \varepsilon=0.005, d=1.1\}$ in this paper. A high number of data sets assures accurate results at the expanse of computational efficiency.

The pseudo-code about the procedure of subtractive clustering is described in Algorithm 2.

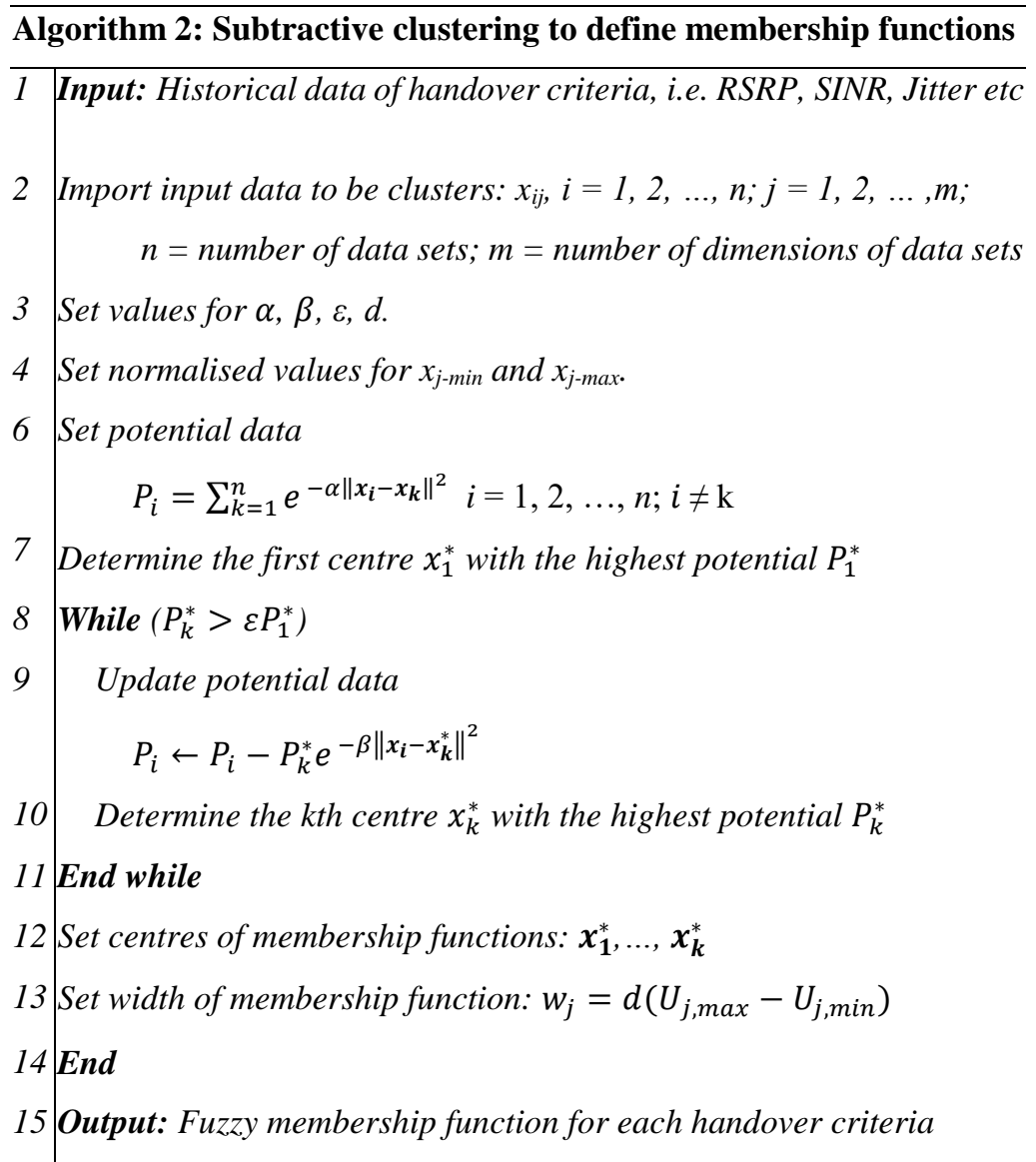

\subsection{Initialisation-Generate decision matrix and weight array}

As defined in 3GPP [3], the radio resource control (RRC) in UE will initiate the measurement for neighbouring BS when its RSRP is lower than a threshold "s-Measure". In Fuzzy-TOPSIS handover scheme, at the time step t, the measured network parameters (criteria $C_{\mathrm{j}}$ ) such as RSRP, SINR, Jitter etc. from each BS (alternatives $A_{\mathrm{i}}$ ) will be fed into a decision matrix $D M^{t}$, as shown in Eq. (21). 


$$
\begin{aligned}
& \operatorname{RSRP}^{t} \text { SINR }^{t} \text { Jitter }^{t} \ldots \quad \ldots \quad C_{n}^{t} \\
& D M^{t}=\begin{array}{c|cccccc}
B S_{1} & x_{11}^{t} & x_{12}^{t} & x_{13}^{t} & \ldots & \ldots & x_{1 n}^{t} \\
B S_{2} & x_{21}^{t} & x_{22}^{t} & x_{23}^{t} & \ldots & \ldots & x_{2 n}^{t} \\
\vdots & \vdots & \vdots & \vdots & \ddots & \ddots & \vdots \\
\vdots & \vdots & \vdots & \vdots & \ddots & \ddots & \vdots \\
B S_{m} & x_{m 1}^{t} & x_{m 2}^{t} & x_{m 3}^{t} & \ldots & \ldots & x_{m n}^{t}
\end{array} \mid
\end{aligned}
$$

where each row in this matrix represent on candidate $\mathrm{BS}$, and each column perform one criterion; $\mathrm{t} \in$ $\{1, \ldots, T\}$, and $\mathrm{T}$ is the fixed time period for mobility observations; $x_{i j}^{t}$ in DM represents the value of $\mathrm{j}^{\text {th }}$ criteria $C_{\mathrm{j}}(\mathrm{j} \in\{1, \ldots, n\})$ of the $i^{\text {th }}$ alternatives $\mathrm{A}_{\mathrm{i}}(\mathrm{i} \in\{1, \ldots, m\})$ at the time period $t$. For instance, the first raw alternatives $A_{1}$ represents candidate $\mathrm{BS}_{1}$ have $\mathrm{n}$ network parameters as criteria, the value of each criteria at the time step $t$ described by $x_{11}^{t}$ to $x_{1 n}^{t}$. And the content in $D M^{t}$ will be normalised by Eq. (8) and (9) in order to calculate weight value for each criterion.

The weighted value of each criterion can be determined by either by subjective or objective weighing approach. To avoid human interference, an objective weighting method - entropy is utilised to calculate weight value $w_{j}$ of each criterion as shown in Eqs. (22) and (23). The entropy is originally derived from the concepts of thermodynamics and it measures the amount of instability in a system. For the content in decision matrix, higher entropy indicates more information is contained in a criterion, and thus greater weight.

$$
\begin{gathered}
w_{j}=\frac{1-E_{j}}{\sum_{j=1}^{m}\left(1-E_{j}\right)} \\
E_{j}=-\frac{\sum_{i=1}^{n}\left(x_{i j} * \ln \left(x_{i j}\right)\right)}{\ln (n)}
\end{gathered}
$$

After normalisation and weight calculation for each alternative, the weight array for each criterion can hence obtain as,

$$
W^{t}=\left[w_{1}^{t}, w_{2}^{t}, \ldots \ldots w_{n}^{t}\right]
$$

\subsection{Decision-Making by fuzzy-TOPSIS engine}

The conventional MADM approach cannot process RRM parameters that are under the influence of uncertain and imprecise data, especially in the presence of interference and noise. Inherently, conventional MADM is not able to optimise the performance of the handover algorithm. Hence, the fuzzy-TOPSIS are more suitable in handover decision making to obtain a more accurate decision, as discussed in Section 2. 
In the fuzzy-TOPSIS approach, all of the content in the normalised decision matrix and weight array will be transformed into a fuzzy matrix $\widetilde{D M}(25)$ and array $\widetilde{W}(26)$ by mapping into the fuzzy membership functions that defined at the initialisation stage.

$$
\begin{aligned}
& \operatorname{RSRP}^{t} \operatorname{SINR}^{t} \text { Jitter }^{t} \ldots \quad \ldots \quad C_{n}^{t} \\
& \widetilde{D M}^{t}=\begin{array}{c|cccccc}
B S_{1} & \tilde{x}_{11}^{t} & \tilde{x}_{12}^{t} & \tilde{x}_{13}^{t} & \ldots & \ldots & \tilde{x}_{14}^{t} \\
B S_{2} & \tilde{x}_{21}^{t} & \tilde{x}_{22}^{t} & \tilde{x}_{23}^{t} & \ldots & \ldots & \tilde{x}_{24}^{t} \\
\vdots & \vdots & \vdots & \vdots & \ddots & \ddots & \vdots \\
\vdots & \vdots & \vdots & \vdots & \ddots & \ddots & \vdots \\
B S_{m} & \tilde{x}_{1 m}^{t} & \tilde{x}_{2 m}^{t} & \tilde{x}_{3 m}^{t} & \ldots & \ldots & \tilde{x}_{4 m}^{t}
\end{array} \\
& \widetilde{W}^{t}=\left[\widetilde{w}_{1}^{t}, \widetilde{w}_{2}^{t}, \ldots \ldots \widetilde{w}_{n}^{t}\right]
\end{aligned}
$$

In the fuzzy decision matrix $\widetilde{D M}, \tilde{x}_{i j}^{t}=\left(a_{i j}, b_{i j}, c_{i j}\right)$ represents the triangular fuzzy number of $j^{\text {th }}$ handover criteria of $i^{\text {th }}$ neighbouring BS at the time step $t$; the degree of importance for each decision criteria at the time step $t$ is represented by $\widetilde{w}_{j}^{t}=\left(a_{j}, b_{j}, c_{j}\right)$ in weight array $\widetilde{W}^{t}$. Afterwards, the $\widetilde{D M}$ will multiply with $\widetilde{\mathrm{W}}$ to calculate weighted fuzzy decision matrix $\widetilde{V}$ as,

$$
\begin{aligned}
\tilde{V}^{t}=\left|\begin{array}{cccccc}
\tilde{v}_{11}^{t} & \tilde{v}_{12}^{t} & \tilde{v}_{13}^{t} & \ldots & \ldots & \tilde{v}_{1 n}^{t} \\
\tilde{v}_{21}^{t} & \tilde{v}_{22}^{t} & \tilde{v}_{23}^{t} & \ldots & \ldots & \tilde{v}_{2 n}^{t} \\
\vdots & \vdots & \vdots & \ddots & \ddots & \vdots \\
\vdots & \vdots & \vdots & \ddots & \ddots & \vdots \\
\tilde{v}_{m 1}^{t} & \tilde{v}_{m 2}^{t} & \tilde{v}_{m 3}^{t} & \ldots & \ldots & \tilde{v}_{m n}^{t}
\end{array}\right| \\
=\left|\begin{array}{ccccccc}
\tilde{w}_{1}^{t} \tilde{x}_{11}^{t} & \widetilde{w}_{2}^{t} \tilde{x}_{12}^{t} & \widetilde{w}_{3}^{t} \tilde{x}_{13}^{t} & \ldots & \ldots & \widetilde{w}_{n}^{t} \tilde{x}_{1 n}^{t} \\
\widetilde{w}_{1}^{t} \tilde{x}_{21}^{t} & \widetilde{w}_{2}^{t} \tilde{x}_{22}^{t} & \widetilde{w}_{3}^{t} \tilde{x}_{23}^{t} & \ldots & \ldots & \widetilde{w}_{n}^{t} \tilde{x}_{2 n}^{t} \\
\vdots & \vdots & \vdots & \ddots & \ddots & \vdots \\
\vdots & \vdots & \vdots & \ddots & \ddots & \vdots \\
\widetilde{w}_{1}^{t} \tilde{x}_{m 1}^{t} & \widetilde{w}_{2}^{t} \tilde{x}_{m 2}^{t} & \widetilde{w}_{3}^{t} \tilde{x}_{m 3}^{t} & \ldots & \ldots & \widetilde{w}_{n}^{t} \tilde{x}_{m n}^{t}
\end{array}\right|
\end{aligned}
$$

Afterwards, the normalised weighted fuzzy decision matrix $\widetilde{V}$ will be feed into fuzzy-TOPSIS decision engine to obtain the fuzzy PIS $\left(A^{+}\right)$and fuzzy NIS $\left(A^{-}\right)$by Eqs. (28) and (29),

$$
\begin{aligned}
& A^{+}=\widetilde{V}_{J}^{+}(j=1,2, \ldots, m) \quad \text { where } \widetilde{V}_{J}^{+}=\max _{i} \widetilde{V_{l \jmath}} \\
& A^{-}=\widetilde{V}_{J}^{-}(j=1,2, \ldots, m) \quad \text { where } \widetilde{V}_{J}^{-}=\min _{i} \widetilde{V_{l \jmath}}
\end{aligned}
$$

From $A^{+}$and $A^{-}$, the closeness between each candidate BSs to both fuzzy PIS and fuzzy NIS can be calculated by Eqs. (6) and (30) -(31),

$$
\begin{aligned}
& d_{i}^{+}=\sum_{j=1}^{n} d\left(\widetilde{V_{l \jmath}}, \widetilde{V}_{J}^{+}\right) \\
& d_{i}^{-}=\sum_{j=1}^{n} d\left(\widetilde{V_{l \jmath}}, \widetilde{V}_{J}^{-}\right)
\end{aligned}
$$


Finally, the closeness coefficient $C C_{\mathrm{i}}$ of each candidate BSs can be calculated by Eq. (14). Where, the closeness coefficient $C C_{\mathrm{i}}$ will be used to trigger handover process and select target $\mathrm{BS}$ at the next stage. The pseudo code for fuzzy-TOPSIS decision engine is summarised as given in Algorithm 3.

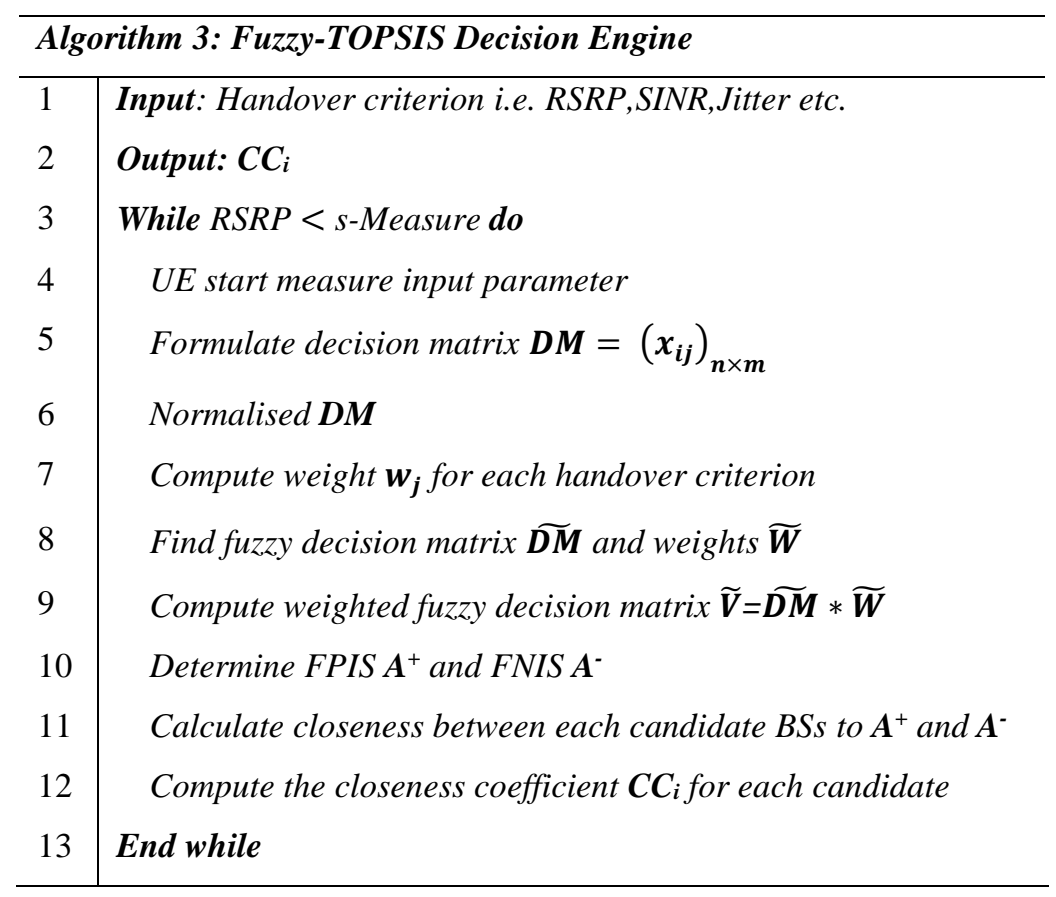

\subsection{Handover triggering and target selection}

The conventional handover mechanism is usually based on a single attribute to make decisions. The single attribute based triggering mechanisms are easily affected by interference and thus causing effects like ping-pong handover, frequent handover and unnecessary handover. Besides, most of the existing triggering approach only considers RRM conditions of the serving BS while ignoring the neighbouring BSs.

To overcome the limitation of the existing approach, this paper adopts $C C$ from TOPSIS as the key factor to determine the triggering time and handover target. As mentioned earlier, the outcome of TOPSIS decision engine, $C C$, represents the similarities between each candidate $\mathrm{BS}$ to the ideal solution. In other words, the candidate with the highest $C C$ would be the best option to handover amongst all available candidates. When deciding on the $B S_{\text {best }}$ with the highest $C C$ ( $\left.C C_{\text {highest }}\right)$, the proposed scheme will compare $C C$ of the UE's serving BS ( $\left.C C_{\text {serving }}\right)$ with $C C_{\text {highest. }}$ The UE will trigger and send handover request to serving $\mathrm{BS}$ only when the ratio $C C_{\text {serving }} / C C_{\text {highest }}$ lower than 0.75 . Otherwise, the UE will maintain the connection with the current serving BS until the triggering condition is fulfilled. Here, the 
0.75 is seen the best $C C_{\text {serving }} / C C_{\text {highest }}$ value that could balance a number of handovers, handover pingpong ratio and QoS at an optimal level. This value is obtained as a result of many rounds of simulations with different random seed values. The pseudo code of this triggering and selection mechanism is as shown in Algorithm 4.

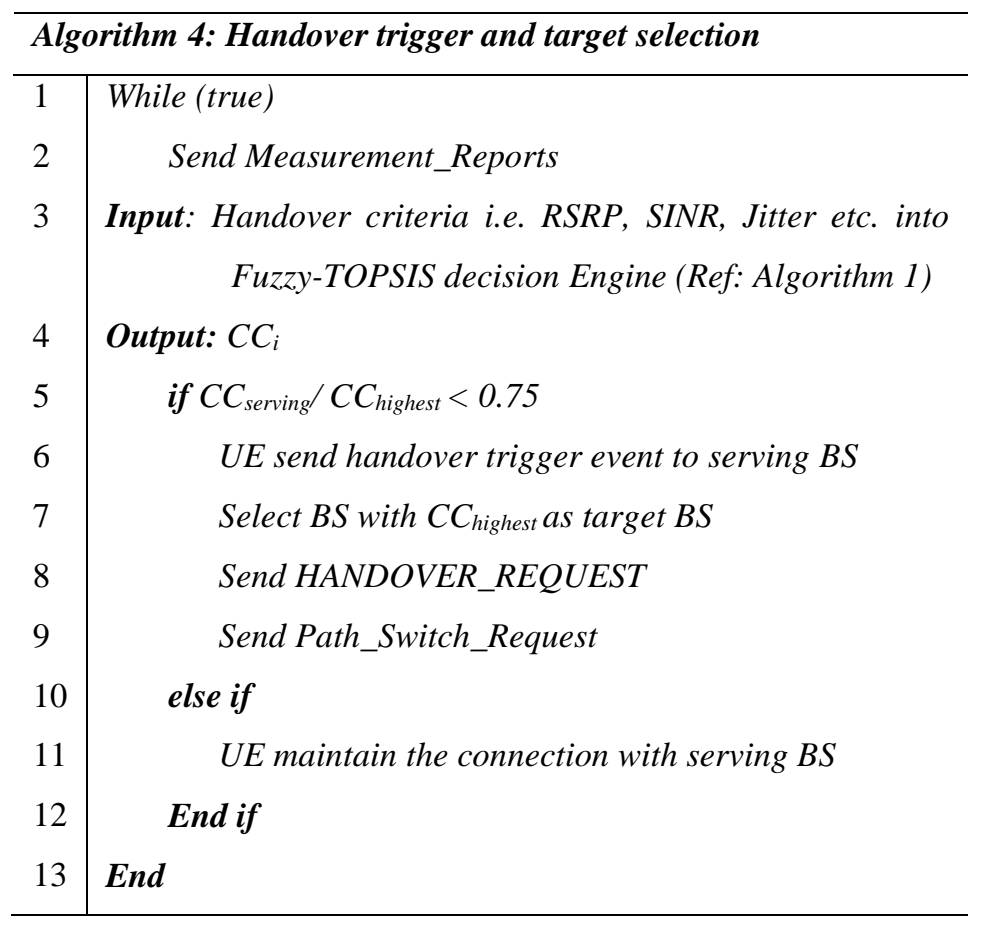

\section{Performance evaluation}

In this section, the evaluation of the proposed fuzzy clustering based TOPSIS algorithm is presented in both numerical and simulation results. The traditional received signal strength (RSS)-based handover scheme and MADM scheme are also simulated for comparison.

\subsection{Numerical analysis}

Consider there are three BSs in the environment, and each BS have three RRM parameter, i.e. RSRP, SINR and jitter, while the $B S_{l}$ is the serving BS of UE. At the time $T$, the collected data from UE can construct a decision matrix $D M$ as,

$$
\begin{aligned}
& \text { RSRP SINR Jitter }
\end{aligned}
$$

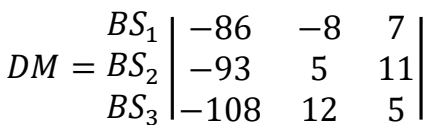


All content in this matrix will be normalised by using Eqs. (8) and (9), where RSRP and SINR are considered as benefit criteria, and jitter is cost criteria. Then, the weight value of each criterion can be calculated by Eqs. (22) and (23), as in Table 1,

The normalised decision matrix and weight array will be mapped into membership functions and then transformed into fuzzy triangular membership number as in Table 2. To simplify, the generalised fuzzy membership functions (see Fig.2) are used in the numerical example.

Table 1 Normalised decision matrix and weight array

\begin{tabular}{l|lll}
\hline & RSRP & SINR & Jitter \\
\hline$B S_{1}$ & 1 & 0 & 0.67 \\
$B S_{2}$ & 0.68 & 0.65 & 0 \\
$B S_{3}$ & 0 & 1 & 1 \\
$W$ & 0.33 & 0.33 & 0.33 \\
\hline
\end{tabular}

Table 2 Fuzzy triangular number for each criterion and weight value

\begin{tabular}{c|ccc}
\hline & RSRP & SINR & Jitter \\
\hline$B S_{1}$ & $(0.75,0.90,1.00)$ & $(0.00,0.10,0.25)$ & $(0.55,0.70,0.85)$ \\
$B S_{2}$ & $(0.55,0.70,0.85)$ & $(0.55,0.70,0.85)$ & $(0.00,0.10,0.25)$ \\
$B S_{3}$ & $(0.00,0.10,0.25)$ & $(0.75,0.90,1.00)$ & $(0.75,0.90,1.00)$ \\
$W$ & $(0.15,0.30,0.45)$ & $(0.15,0.30,0.45)$ & $(0.15,0.30,0.45)$ \\
\hline
\end{tabular}

Table 3 Fuzzy weighted decision matrix $\widetilde{V}$

\begin{tabular}{c|ccc}
\hline & RSRP & SINR & Jitter \\
\hline$B S_{1}$ & $(0.113,0.27,0.45)$ & $(0.00,0.03,0.113)$ & $(0.083,0.21,0.383)$ \\
$B S_{2}$ & $(0.083,0.21,0.383)$ & $(0.083,0.21,0.383)$ & $(0.00,0.03,0.113)$ \\
$B S_{3}$ & $(0.00,0.03,0.113)$ & $(0.113,0.27,0.45)$ & $(0.113,0.27,0.45)$ \\
\hline
\end{tabular}

Table 4 Analysis of fuzzy TOPSIS

\begin{tabular}{c|cccccc}
\hline & RSRP & SINR & Jitter & $d_{i}^{+}$ & $d_{i}^{-}$ & $C C_{i}$ \\
\hline$B S_{1}$ & $(0.113,0.27,0.45)$ & $(0.00,0.03,0.113)$ & $(0.083,0.21,0.383)$ & 0.30 & 0.44 & 0.59 \\
$B S_{2}$ & $(0.083,0.21,0.383)$ & $(0.083,0.21,0.383)$ & $(0.113,0.27,0.45)$ & 0.36 & 0.39 & 0.52 \\
$B S_{3}$ & $(0.00,0.03,0.113)$ & $(0.113,0.27,0.45)$ & $(0.00,0.03,0.113)$ & 0.25 & 0.50 & 0.67 \\
$A^{+}$ & $(0.113,0.27,0.45)$ & $(0.113,0.27,0.45)$ & $(0.113,0.27,0.45)$ & & & \\
$A^{-}$ & $(0.00,0.03,0.113)$ & $(0.00,0.03,0.113)$ & $(0.00,0.03,0.113)$ & & & \\
\hline
\end{tabular}


From Table 2, the fuzzy decision matrix $\tilde{V}$ can hence obtain by multiplying criteria with each weight value by Eq. (5),

Afterwards, the fuzzy PIS $\left(A^{+}\right)$and fuzzy NIS $\left(A^{-}\right)$can be calculated by Eqs. (28) and (29) from Table 3. And the distance from each alternative to fuzzy PIS $\left(d_{i}^{+}\right)$and fuzzy NIS $\left(d_{i}^{-}\right)$, as well as the closeness coefficient (CC) to the ideal solution are then be calculated by Eqs. (30), (31) and (14) as shown in Table 4.

According to the numerical results in Table 4, the $\mathrm{BS}_{3}$ with the highest $C C$ is 0.67 and hence be selected as the optimal handover target. Meanwhile, the $C C$ of serving BS of UE, $C C_{1}$ is 0.59 . Due to the $\mathrm{CC}_{1} / \mathrm{CC}_{\max }$ ratio of 0.88 , which higher than the triggering ratio (i.e. 0.75 ), and the system will choose to maintain the connection of UE with $\mathrm{BS}_{1}$.

In the next time step $t+1$, the proposed scheme will repeat these procedures until $C C_{\text {serving }} / C C_{\max }$ less than 0.75 , and then handover process will be triggered and switch UE's connection to the BS with the highest $C C$. This scheme will turn off when RSRP higher than the system measuring threshold.

\subsection{Scenario configuration}

A simulation model has been developed in MATLAB to evaluate the effectiveness of the proposed handover scheme. The simulation setup is presented in Table 5. There are 16 BSs are deployed in a $1000 \mathrm{~m} \times 1000 \mathrm{~m}$ scenario, and the distance between each of them is $400 \mathrm{~m}$. The COST-Hata model is used to model the radio propagation of each BSs. The additive white Gaussian noise (AWGN) and Rayleigh noise are added to the transmitted signal. There are 40 UEs will randomly move at this scenario with a constant speed of $30 \mathrm{~km} / \mathrm{h}$.

The RSRP, SINR, packet loss ratio and network jitter are used as handover criterion in this paper. The number of handovers, ping-ping handover ratio and mean option score (MOS) is used as performance indicators to evaluate the performance of the proposed algorithm. The conventional RSS-based handover algorithm, tional SAW and TOPSIS based handover algorithm, and fuzzy-TOPSIS algorithm with generalised fuzzy membership function are used to compare with the proposed scheme. The ping-pong handover in this paper is defined as when UE handed back to the same serving BS within 5s. The pingpong handover ratio is calculated as (32), 


$$
\text { Pingpong hadnover ratio }(\%)=\frac{\text { Number of Pingpong hadnover }}{\text { Number of handover }}
$$

The MOS is used to represent QoS with a scale from '5' (best) to ' 1 ' (worst). Traditionally, the values of MOS is determined using subjective approach. However, ITU-T recommendation G.107 [27], also known as the E-Model, specifies a more objective based evaluation based on measurement of network parameters such as jitter and packet loss ratio. To ensure a promising evaluation result, each KPI will be evaluated at least 100 times to obtain statistically significant results.

\subsection{Results and Discussion}

Fig.4 depicts the membership functions generated by the subtractive clustering method for different criteria, and Fig.5 indicates the probability density function of 16000 input data points for each criterion. An apparent relationship can be found between the membership functions and the corresponding probability density functions. The probability density functions of RSRP and SINR are similar to Gaussian distribution with mean value at around 0.4 and 0.6. The membership functions for these two criteria are hence more concentrated on the corresponding mean values rather than spread evenly over the input space.

Table.5. Simulation parameter

\begin{tabular}{l|l}
\hline Parameters & Specification \\
\hline BS transmitted power: & $40 \sim 45 \mathrm{dBm}$ \\
Carrier frequency: & $1.5 \sim 2 \mathrm{GHz}$ \\
Duration of simulation & $10000 \mathrm{~s}$ \\
Mobility model & Random direction \\
Number of BSs & 16 \\
The distance between each BS & $400 \mathrm{~m}$ \\
Number of UE & 40 \\
UE speed & $30 \mathrm{~km} / \mathrm{h}$ \\
Propagation model: & COST-Hata model \\
Type of Noise & AWGN, Rayleigh \\
\hline
\end{tabular}



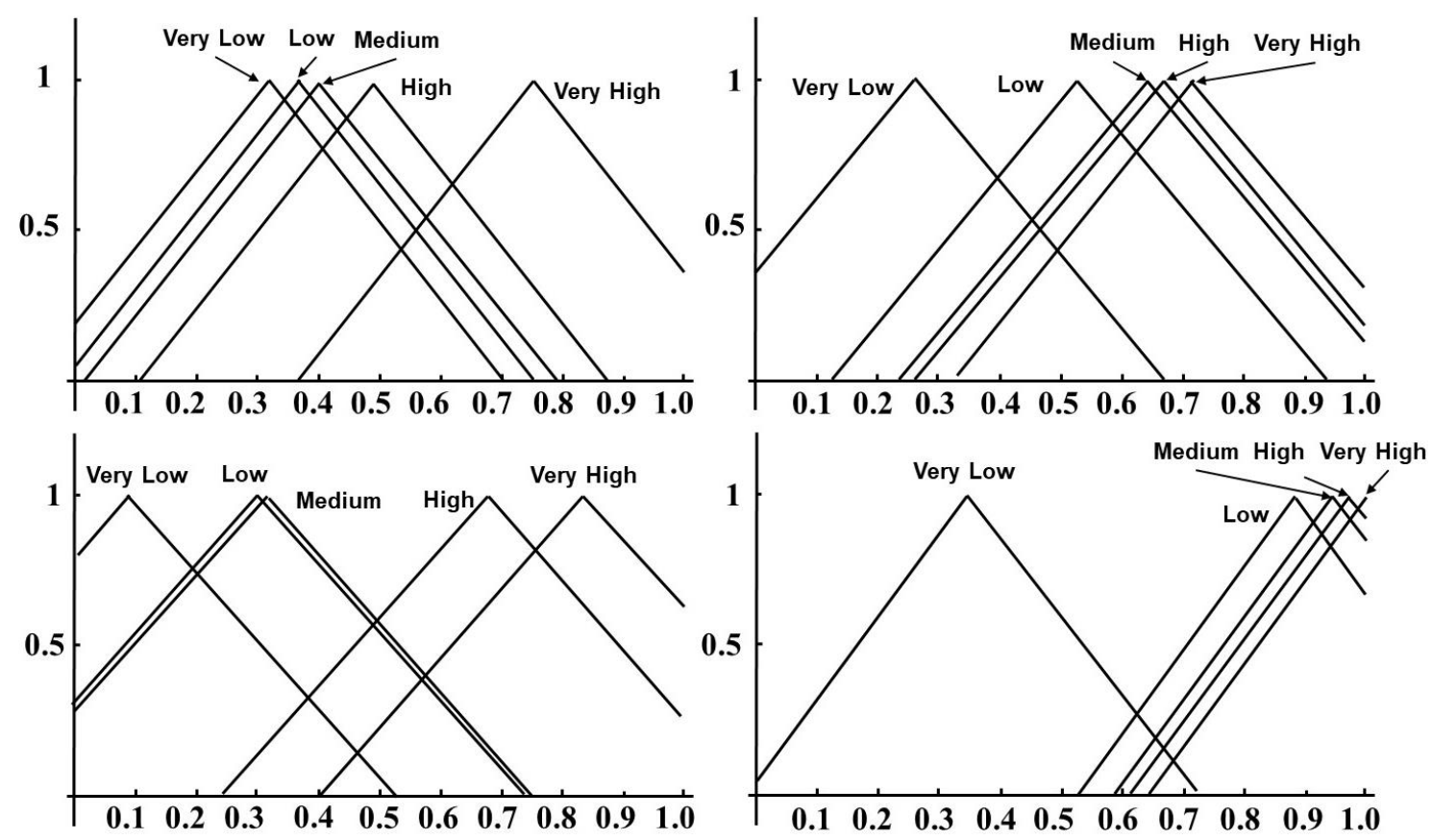

Fig. 4. Membership generated by subtractive clustering for RSRP (top left), SINR (top right), Packet loss rate (bottom left) and network jitter (bottom right)
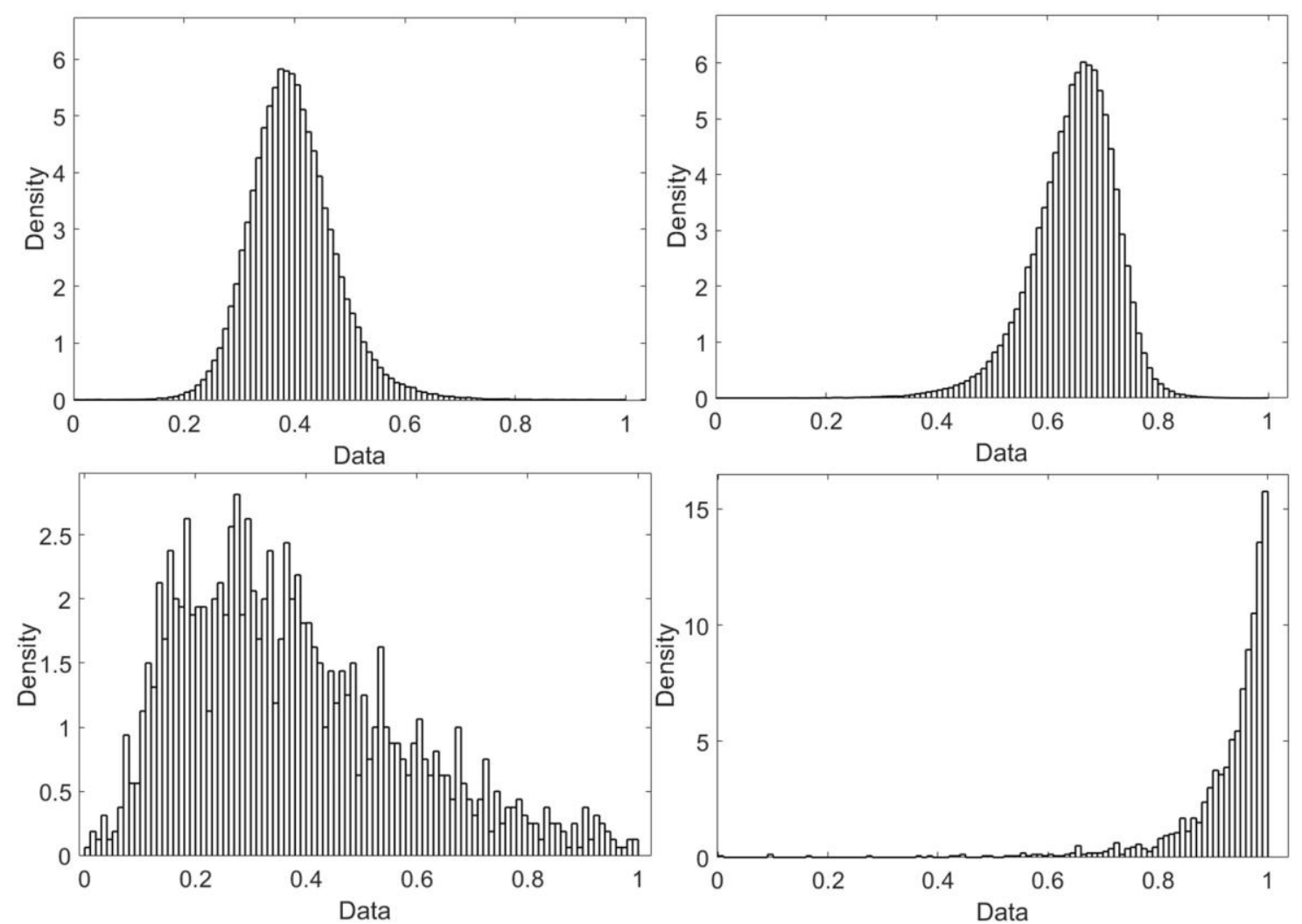

Fig. 5. PDFs by subtractive clustering for RSRP (top left), SINR (top right), Packet loss rate (bottom left) and

$$
\text { Network jitter (bottom right) }
$$

The probability density function of packet loss ratio also follows a distribution with a mean at around 0.3 but with a lower peak value compared with RSRP and SINR, which results in a more scattered 
allocation of the membership functions. The network jitter is modelled by a Pareto distribution. Since jitter is a cost criterion in this work, the normalisation step ends up with an input data following an inverse Pareto distribution as in Fig.5, where an extremely high concentration can be found around 0.97. The membership functions for jitter hence focus on the end of the input space. According to the results in Fig.4 and 5, the proposed subtractive clustering approach can effectively extract the feature of input data and then generate the optimal fuzzy membership functions according to the feature of each criterion. By applying this method into fuzzy-TOPSIS decision engine, the subjective error in defining membership functions can, therefore, be eliminated. This will further enhance the performance of fuzzy-TOPSIS. In practice, the maintenance and optimisation costs of the proposed handover scheme can also be saved due to the characteristics of subtractive clustering.

As indicated in Fig.6-8, the convention RSS-based handover algorithm has the highest number of handovers, ping-pong ratio and the lowest MOS. Due to RSS-based is a single attribute based algorithm, the triggering decision and handover target are only determined by RSRP. The RSRP is fluctuated heavily due to the existing of noise, which will be easily affected by interference. This will render the handover to become abnormal and result in the worst performance. On the other hand, the conventional MADM approach, i.e. SAW and TOPSIS, can consider more than one attribute as decision criteria. From the results, these two approaches have close to similar performance in terms of the number of handovers, Ping-Pong handover ratio and MOS. Although the performance of these two methods is better than the RSS-based handover scheme, it is still lower than fuzzy-TOPSIS with generalised membership functions and fuzzy-TOPSIS with clustering approach.

The fuzzy-TOPSIS with generalised membership function used membership functions as depicted in Fig.2 during the fuzzification stage. The simulation results show the overall performance of this method is better than the previous approach. It can reduce the number of handovers significantly by almost $90 \%$ and ping-pong handover ratio by $10 \%$ as compared to the RSS-based approach. Furthermore, this approach can obtain a relatively high MOS while maintaining a minimum of number of handovers. Finally, the proposed fuzzy-TOPSIS with modified membership function in this paper has the best performance amongst all approaches. This approach can reduce another $10 \%$ number of handovers and $3 \%$ of ping-pong handover ratio by comparing with fuzzy-TOPSIS with generalised membership function. Moreover, the proposed scheme can also maintain QoS in a relative high MOS. 
According to the evaluation above, the proposed scheme outperforms the traditional approach in terms of number of handovers, ping-pong handover ratio and MOS. By comparing with the other traditional approach, the proposed handover scheme has the following advantages. Firstly, the proposed scheme integrated both the advantages of fuzzy logic and MADM techniques. With the feature of MADM, the proposed scheme can effectivity deal with multiple criteria and make an optimal decision. And the feature of fuzzy membership functions can enable the proposed scheme to process uncertain and imprecise data, and thus minimise the effect of environmental noise and interference. Secondly, the membership functions generated by subtractive clustering for each input criteria are further enhancing the performance of the proposed scheme. The membership functions generated by subtractive clustering can effectivity represent the actual feature of input data, and hence can transform each data into the right membership function. Thirdly, the implementation of entropy to calculate weight value for each criterion can eliminate the human error, and present more objective results. With the above characteristics, the proposed handover scheme can select an optimal BS among all candidates and criteria. On top of that, this paper utilises closeness coefficient $C C$ from the proposed algorithm to trigger handover. This novel triggering mechanism allows BS to decide a triggering timing based on the overall performance of the application scenario rather than only consider the RRM condition of UE's serving BS. Therefore, it can further minimise the number of handovers and ping-pong handover ratio.

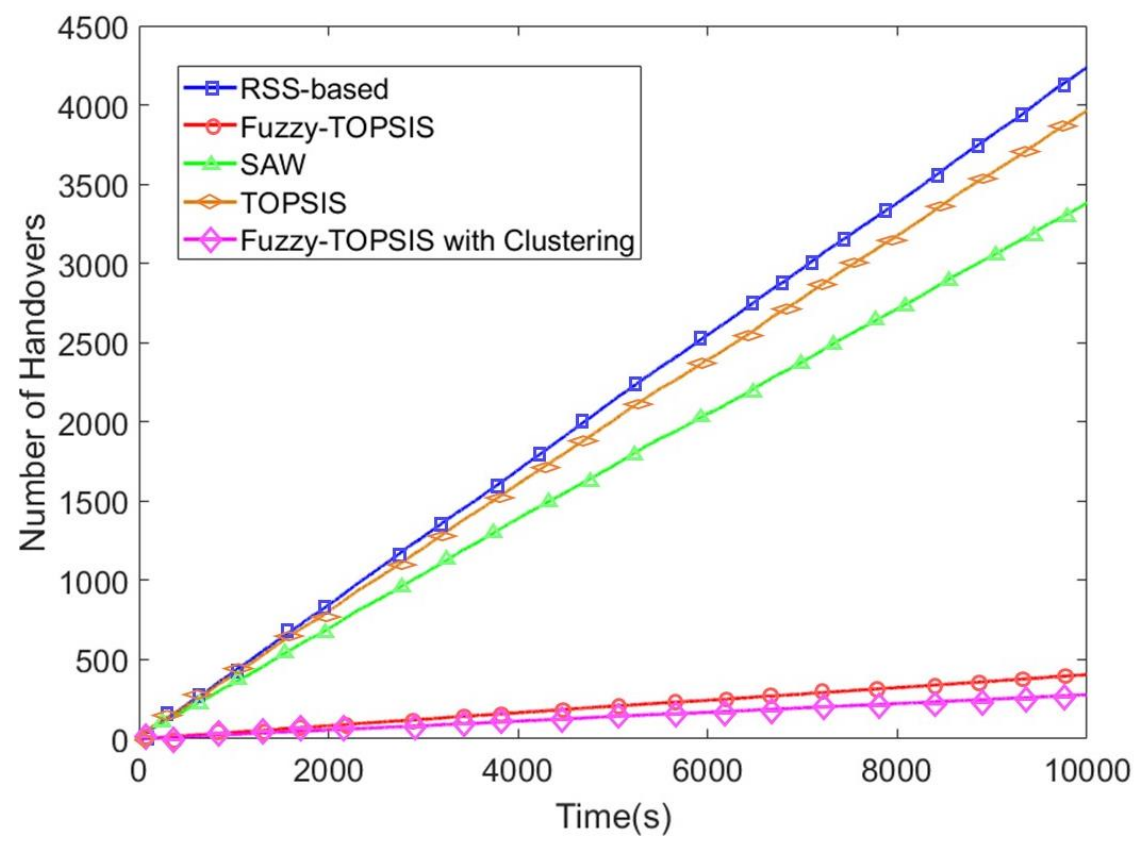

Fig. 6. Number of handovers by different approaches 


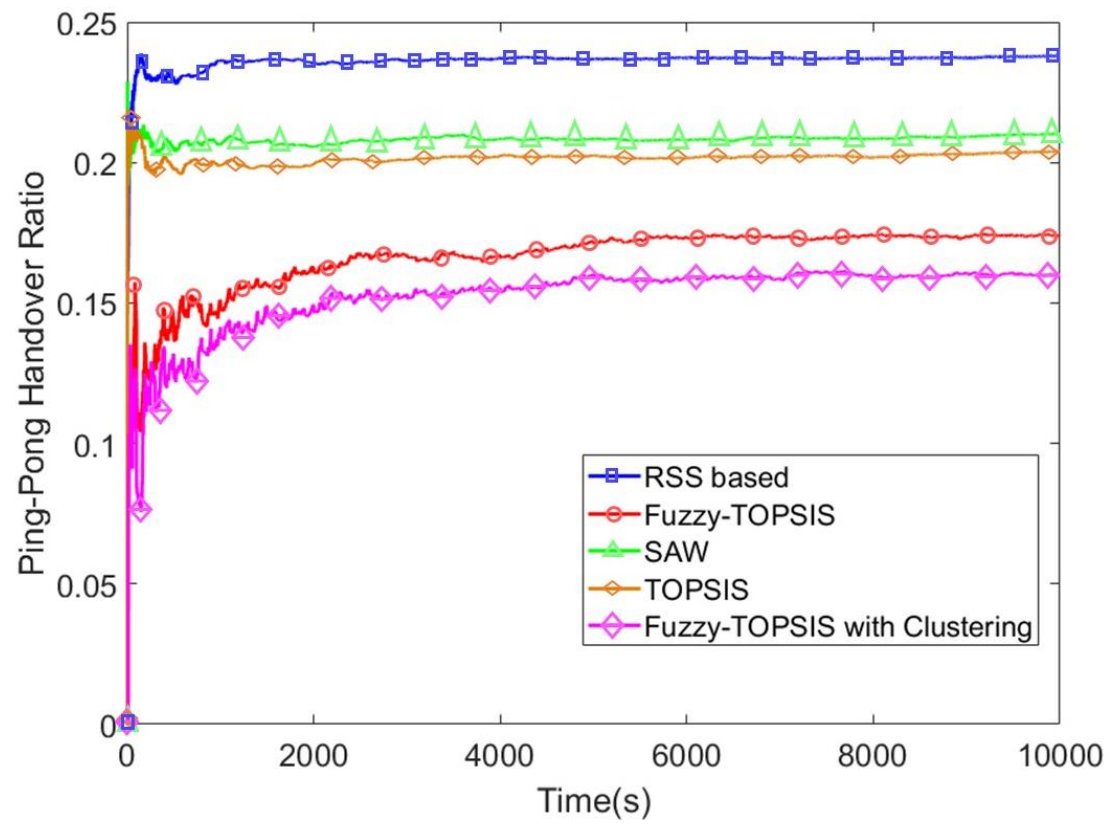

Fig. 7. Handover ping-pong ratio by different approaches

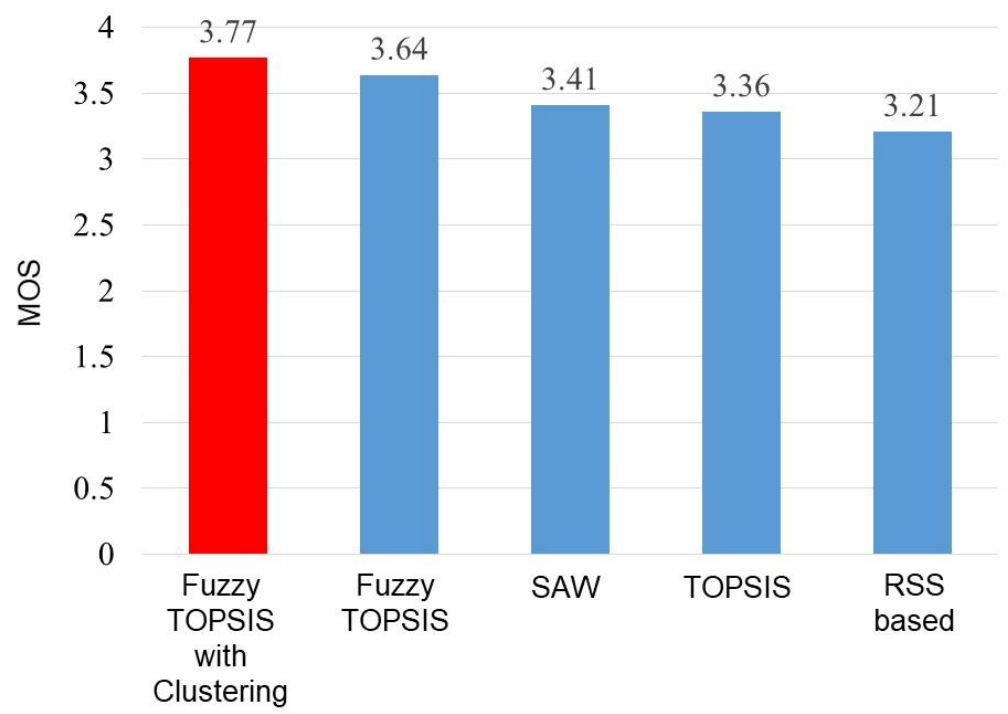

Fig. 8. MOS from different approaches

\section{Conclusion}

To minimise the challenges of handover in 5G-UDN and also overcome the limitation of the existing handover approach, the work presented in this paper developed a fuzzy-TOPSIS based handover algorithm by considering more than one attribute as criteria to trigger handover process and select optimal neighbouring BSs as handover target. The proposed method combines the strengths of both TOPSIS and fuzzy logic. To further enhance the performance of the proposed scheme. We proposed the use of 
subtracting clustering scheme to intelligent generate fuzzy membership function by using historical data.

The simulation results indicated that the proposed handover scheme could significantly reduce the number of handovers and ping-pong handover ratio while retaining QoS at a relatively high level by comparing with the RSS-based algorithm and conventional MADM approach. And the proposed subtracting clustering technique can intelligently define the optimal fuzzy membership function from historical data for different criterion. Base on this optimisation, the proposed scheme with customised membership function outperforms fuzzy-TOPSIS approach with generalised fuzzy membership function in terms of all evaluated aspects.

\section{Acknowledgement}

The authors acknowledge the financial support from the International Doctoral Innovation Centre (IDIC), Ningbo Education Bureau, Ningbo Science and Technology Bureau, and the University of Nottingham.

This work was also supported by Ningbo Natural Science Programme, project code 2018A610095.

\section{Reference}

[1] Cisco, "Cisco Visual Networking Index: Global Mobile Data Traffic Forecast Update, 2017-2022 White Paper," San Jose, CA, USA, 2019.

[2] the 3GPP Organizational Partners, Evolved Universal Terrestrial Radio Access (E-UTRA),Radio Resource Control (RRC),Protocol specification,document TS 36.331. 2018.

[3] the 3GPP Organizational Partners, Radio Resource Control (RRC) protocol specification, document TS 38.331. 2018

[4] K. Da Costa Silva, Z. Becvar, and C. R. L. Frances, “Adaptive Hysteresis Margin Based on Fuzzy Logic for Handover in Mobile Networks with Dense Small Cells," IEEE Access, vol. 6, no. c, pp. 17178-17189, 2018.

[5] K. Vasudeva, S. Dikmese, I. Güvenç, A. Mehbodniya, W. Saad, and F. Adachi, "Fuzzy-Based Game Theoretic Mobility Management for Energy Efficient Operation in HetNets," IEEE Access, vol. 5, pp. 7542-7552, 2017.

[6] I. De La Bandera, P. Munoz, I. Serrano, and R. Barco, “Adaptive Cell Outage Compensation in SelfOrganizing Networks,” IEEE Trans. Veh. Technol., vol. 67, no. 6, pp. 5231-5244, 2018.

[7] A. M. Aibinu, A. J. Onumanyi, A. P. Adedigba, M. Ipinyomi, T. A. Folorunso, and M. J. E. Salami, "Development of hybrid artificial intelligent based handover decision algorithm," Eng. Sci. Technol. an Int. J., vol. 20, no. 2, pp. 381-390, 2017.

[8] H. Lu, Y. Li, S. Mu, D. Wang, H. Kim, and S. Serikawa, "Motor anomaly detection for unmanned aerial vehicles using reinforcement learning," IEEE Internet Things J., vol. 5, no. 4, pp. 2315-2322, 2018.

[9] H. Lu, Q. Liu, D. Tian, Y. Li, H. Kim, and S. Serikawa, "The Cognitive Internet of Vehicles for Autonomous Driving,” IEEE Netw., vol. 33, no. 3, pp. 65-73, 2019.

[10] Y. Zhang, R. Gravina, H. Lu, M. Villari, and G. Fortino, "PEA: Parallel electrocardiogram-based authentication for smart healthcare systems," J. Netw. Comput. Appl., vol. 117, pp. 10-16, 2018.

[11] H. Lu, Y. Li, M. Chen, H. Kim, and S. Serikawa, "Brain Intelligence: Go beyond Artificial Intelligence," Mob. Networks Appl., vol. 23, no. 2, pp. 368-375, 2018.

[12] H. Lu et al., "CONet : A Cognitive Ocean Network," IEEE Wirel. Commun., 2019.

[13] C. F. Kwong, T. C. Chuah, S. W. Tan, and A. Akbari-Moghanjoughi, "An adaptive fuzzy handover triggering approach for Long-Term Evolution network,” Expert Syst., vol. 33, no. 1, pp. 30-45, 2016. 
[14] B. R. Chandavarkar and R. M. R. Guddeti, "Simplified and improved multiple attributes alternate ranking method for vertical handover decision in heterogeneous wireless networks," Comput. Commun., vol. 83, pp. 81-97, 2016.

[15] C. H. F. Dos Santos, M. P. S. De Lima, F. S. Dantas Silva, and A. Neto, "Performance evaluation of multiple attribute mobility decision models: A QoE-efficiency perspective," in International Conference on Wireless and Mobile Computing, Networking and Communications, 2017, vol. 2017Octob.

[16] A. Agrawal, A. Jeyakumar, and N. Pareek, "Comparison between vertical handoff algorithms for heterogeneous wireless networks," Int. Conf. Commun. Signal Process. ICCSP 2016, pp. 1370-1373, 2016.

[17] C.L.Hwang and K.Yoong, "Multiple Attributes Decision Making Methods and Applications," in Springer, Berlin, 1981.

[18] T. Goyal and S. Kaushal, "Handover optimization scheme for LTE-Advance networks based on AHP-TOPSIS and Q-learning,” Comput. Commun., vol. 133, no. September 2018, pp. 67-76, 2019.

[19] A. Habbal, S. I. Goudar, and S. Hassan, "Context-Aware Radio Access Technology Selection in 5G Ultra Dense Networks,” IEEE Access, vol. 5, no. Mmc, pp. 6636-6648, 2017.

[20] M. Alhabo, S. Member, L. Zhang, and S. Member, "Multi-Criteria Handover Using Modified Weighted TOPSIS Methods for Heterogeneous Networks," IEEE Access, vol. PP, no. c, p. 1, 2018.

[21] M. Lahby, A. Attioui, and A. Sekkaki, "An improved policy for network selection decision based on enhanced-topsis and utility function," 2017 13th Int. Wirel. Commun. Mob. Comput. Conf. IWCMC 2017, pp. 2175-2180, 2017.

[22] H. W. Yu and B. Zhang, "A heterogeneous network selection algorithm based on network attribute and user preference," Ad Hoc Networks, vol. 72, pp. 68-80, 2018.

[23] A. Ahmed, L. M. Boulahia, and D. Gaït, "Enabling vertical handover decisions in heterogeneous wireless networks: A state-of-the-art and a classification," IEEE Commun. Surv. Tutorials, vol. 16, no. 2, pp. 776-811, 2014.

[24] S. NĂdǍban, S. Dzitac, and I. Dzitac, "Fuzzy TOPSIS: A General View," Procedia Comput. Sci., vol. 91, no. Itqm, pp. 823-831, 2016.

[25] G. Kabir, M. Ahsan, and A. Hasin, "Comparative Analysis of Topsis and Fuzzy Topsis for the Evaluation of Travel Website Service Quality," vol. 60143, no. 3, pp. 497-11, 2012.

[26] Y. S. Hussein, B. M. Ali, M. F. A. Rasid, A. Sali, and A. M. Mansoor, "A novel cell-selection optimization handover for long-term evolution (LTE) macrocellusing fuzzy TOPSIS," Comput. Commun., vol. 73, pp. 22-33, 2016.

[27] M. A. Raja, A. Jagodzinska, and V. Barriac, “On Losses, Pauses, Jumps, and the Wideband EModel,” IEEE Access, vol. 5, pp. 16130-16148, 2017. 